\title{
Altered glutathione redox state in schizophrenia
}

\author{
Jeffrey K. Yao ${ }^{\mathrm{a}, \mathrm{b}, \mathrm{c}, *}$, Sherry Leonard ${ }^{\mathrm{d}}$ and Ravinder Reddy ${ }^{\mathrm{a}, \mathrm{b}}$ \\ ${ }^{a}$ VA Pittsburgh Healthcare System, 7180 Highland Drive, Pittsburgh, PA 15206, USA \\ ${ }^{\mathrm{b}}$ Department of Psychiatry, Western Psychiatric Institute and Clinic, University of Pittsburgh Medical Center, \\ Pittsburgh, PA 15213, USA \\ ${ }^{\mathrm{c}}$ Department of Pharmaceutical Sciences, School of Pharmacy, University of Pittsburgh, Pittsburgh, PA 15213, \\ USA \\ ${ }^{\mathrm{d}}$ VA Denver Health-care System, Denver, $C O$ and Department of Psychiatry, University of Colorado Health \\ Sciences Center, Denver, CO 80262, USA
}

\begin{abstract}
Altered antioxidant status has been reported in schizophrenia. The glutathione (GSH) redox system is important for reducing oxidative stress. GSH, a radical scavenger, is converted to oxidized glutathione (GSSG) through glutathione peroxidase (GPx), and converted back to GSH by glutathione reductase (GR). Measurements of GSH, GSSG and its related enzymatic reactions are thus important for evaluating the redox and antioxidant status. In the present study, levels of GSH, GSSG, GPx and GR were assessed in the caudate region of postmortem brains from schizophrenic patients and control subjects (with and without other psychiatric disorders). Significantly lower levels of GSH, GPx, and GR were found in schizophrenic group than in control groups without any psychiatric disorders. Concomitantly, a decreased GSH:GSSG ratio was also found in schizophrenic group. Moreover, both GSSG and GR levels were significantly and inversely correlated to age of schizophrenic patients, but not control subjects. No significant differences were found in any GSH redox measures between control subjects and individuals with other types of psychiatric disorders. There were, however, positive correlations between GSH and GPx, GSH and GR, as well as GPx and GR levels in control subjects without psychiatric disorders. These positive correlations suggest a dynamic state is kept in check during the redox coupling under normal conditions. By contrast, lack of such correlations in schizophrenia point to a disturbance of redox coupling mechanisms in the antioxidant defense system, possibly resulting from a decreased level of GSH as well as age-related decreases of GSSG and GR activities.
\end{abstract}

Keywords: Glutathione, glutathione disulfide, glutathione peroxidase, glutathione reductase, cigarette smoking, age, postmortem caudate, schizophrenia

\section{Introduction}

Biological systems have evolved complex protective strategies against free radical toxicity [1]. Under physiological conditions the potential for free radicalmediated damage is kept in check by the antioxidant defense system, comprising a series of enzymatic and

\footnotetext{
${ }^{*}$ Corresponding author: Jeffrey K. Yao, Ph.D., FACB, VA Pittsburgh Healthcare system (Bldg. \#13), 7180 Highland Drive, Pittsburgh, PA 15206, USA. Tel.: +1 412365 4465; Fax: +1 412365 5917; E-mail: jkyao@pitt.edu.
}

non-enzymatic components (Fig. 1). The key antioxidant enzymes include superoxide dismutase (SOD), catalase (CAT), and glutathione peroxidase (GSH-Px). Superoxide dismutase catalyzes the conversion of superoxide radicals $\left(\mathrm{O}_{2}^{-} \bullet\right)$ to hydrogen peroxide $\left(\mathrm{H}_{2} \mathrm{O}_{2}\right)$, which in turn can form the highly reactive hydroxyl radicals. Catalase and glutathione peroxidase convert hydrogen peroxide to water. Glutathione (GSH) is utilized by GSH-Px to yield the oxidized form of glutathione (GSSG), which is converted back to GSH by glutathione reductase (GR). In addition, nitric oxide (NO) which is the product of a five-electron oxidation 


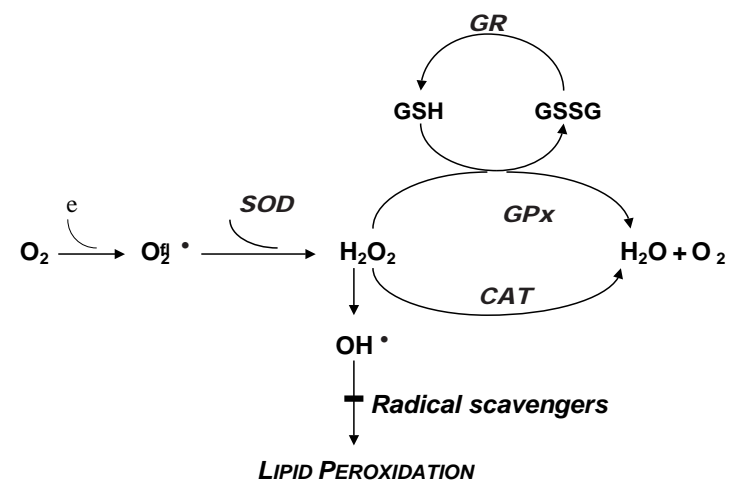

Fig. 1. Production of reactive oxygen species and defense mechanism against damage by reactive oxygen. Abbreviations: $\mathrm{O}_{2}^{-} \bullet$, superoxide radicals; $\mathrm{SOD}$, superoxide dismutase; $\mathrm{H}_{2} \mathrm{O}_{2}$, hydrogen peroxide; CAT, catalase; GPx, glutathione peroxidase; GSH, glutathione; GSSG, glutathione disulfide; GR, glutathione reductase, $\mathrm{OH} \bullet$, hydroxyl radicals.

of the amino acid L-arginine, can also produce hydroxyl radicals as well as nitrogen dioxide radical.

There is evidence that free radicals are involved in membrane pathology, and may play a role in schizophrenia [2-4]. Membrane dysfunction can be secondary to free radical-mediated pathology, and may contribute to specific aspects of schizophrenic symptomatology and complications of its treatment. Specifically, free radical-mediated abnormalities may contribute to the development of a number of clinically significant consequences, including prominent negative symptoms, tardive dyskinesia, neurological "soft" signs, poor premorbid function, and CT scan abnormalities. Our previous results showing altered membrane dynamics and antioxidant enzyme activities in schizophrenia, and findings from other investigators are consistent with the notion of free radical-mediated neurotoxicity in schizophrenia [4]. Free radicals are reactive chemical species generated during normal metabolic processes, and in excess, can damage lipids, proteins, and DNA. Regions of high oxygen consumption, lipid content, and transition metals, are at particular risk. Hence, neuronal membranes are uniquely vulnerable to radical-mediated damage.

Studies of the antioxidant defense system are often complicated by cumbersome analytical methods, which require separate and multi-step extraction and chemical reaction procedures. Thus, measurements of multiple parameters are limited in relatively small biological samples. Recently, we [5] have developed a convenient and sensitive tool to measure low molecular weight, redox-active compounds in biological samples using a high-pressure liquid chromatography sys- tem (HPLC) coupled with a 16-channel Coulometric Multi Electrode Array System (CMEAS). The purpose of the present investigation was to assess the glutathione (GSH) redox system (Fig. 1) by evaluating not only GSH and GSSG levels but also the enzyme activities of GSH-Px and GSH-R simultaneously in the caudate region of postmortem brain samples from patients with schizophrenia as well as controls with and without other psychiatric disorders.

\section{Methods}

\subsection{Postmortem brain tissues}

The Schizophrenia Research Center Brain Bank at the VA Medical Center in Denver, Colorado, provided postmortem brain tissue. Human brain was collected at autopsy from local services following family consent.

Psychiatric illness status was determined independently by two board-certified psychiatrists from medical records and structured family interviews. Diagnostic and Statistical Manual IV criteria for schizophrenia [6] (American Psychiatric Association, 1994), were met for the schizophrenic subjects used in the study. Control subjects were also evaluated and determined to have had no evidence of psychiatric illness.

At autopsy, the brain was weighed and examined for gross pathology. It was then divided sagittally and one hemisphere, selected randomly, was preserved in formalin for neuropathological analysis at the macroscopic and microscopic level. Microscopic evaluations included standard Bielchowsky silver stain on multiple cerebral areas to rule out abnormal neuropathology, such plaques and tangles, associated with Alzheimer's and other conditions. Patients with positive neuritic findings or ambiguous neuropathology reports were excluded from the current study. The hemisphere that was not subjected to neuropathological analysis was sliced coronally into $1 \mathrm{~cm}$ slices, from which multiple regions were dissected in 1-gram blocks, frozen in dry ice snow, and packaged for storage at $-80^{\circ} \mathrm{C}$. Hemispheres are collected randomly.

An extensive review of hospital, autopsy, and neuropathology reports were conducted to determine agonal and postmortem conditions for each subject. Based upon this information, an agonal state score was assigned following the four-point rating scale described by Hardy et al. [7]. These four categories included 1) violent and fast death, almost always of unnatural causes such as shootings (accidental, homicidal, or sui- 
cide) or blunt force trauma; 2) fast death of natural causes, such as the sudden, unexpected deaths of individuals who had appeared reasonably healthy. The most common cause of death in this group was myocardial infarction; 3) intermediate death, usually unexpected, even though the patient was ill prior to death; and 4) slow death, occurring after a long illness. The majority of these patients died of cancer or chronic pulmonary disease.

Additional postmortem parameters, such as age, sex, ethnicity, medication history, psychiatric illness status, cigarette smoking history and alcohol use, were evaluated through hospital, autopsy, and neuropathology reports. Family members and physicians were also interviewed to detail the smoking and alcohol history of the subject, including packs of cigarettes smoked per day, and the quantity and type of alcohol consumed. All of the subjects who had used alcohol prior to death had consumed no more than two drinks per day on average. Smokers had used cigarettes until the time of death.

All patients with schizophrenia and other psychiatric disorders were on antipsychotic medications at the time of death. At the time of autopsy, the brain was removed and hemisectioned in the sagittal plane. One half of the cerebrum, cerebellum, and brainstem was immersed in $10 \%$ formalin and fixed for 1-2 weeks. The other hemisphere was sliced coronally and transported on aluminum plates, previously chilled on ice, to the laboratory for dissection. To avoid hemispheric bias, alternate (left/right) hemispheres were collected. Immediately after sample collection, tissue was dissected from approximately 50 areas, in one gram pieces, and frozen on dry ice snow. The remainder of the hemisphere was also frozen on slabs of dry ice, and stored with the dissected parts at $-80^{\circ} \mathrm{C}$. In the present study, the caudate nucleus from schizophrenic and control groups was used for GSH analyses.

\subsection{Laboratory assays}

\subsubsection{Tissue preparation}

Approximately $0.2 \mathrm{~g}$ of brain sample was first weighed and lyophilized before the dry weight was taken. Samples were then homogenized by a Polytron tissue homogenizer in one $\mathrm{ml}$ of $10 \mathrm{mM}$ Sodium Acetate, $\mathrm{pH}$ 6.5. Following centrifugation for $10 \mathrm{~min}-$ utes at 3,000 rpm, the cell-free homogenate was further passed through a disposable membrane $(0.22 \mu \mathrm{m}$ pore size) micropartition system (Millipore Ultrafree-MC) under centrifugation to remove any compounds above 10,000 nominal molecular weight limit. The resulting protein-free supernatant was subjected to HPLC for GSH and GSSG assays.

\subsubsection{Measurements of GSH and GSSG by HPLC-CMEAS}

The deproteinized sample was analyzed by an ESA CoulArray Model 5600 HPLC system consisting of two Model 582 pumps, one dynamic gradient mixer, two PEEK $^{\mathrm{TM}}$ pulse dampers, a Model 542 refrigerated autosampler injector, a CoulArray organizer module, and a serial array of 16 coulometric electrodes. The system is controlled and chromatograms are analyzed by a Dell personal computer Model Dimension XPS R450 using the ESA CoulArray for Windows-32 software program (version 1.04).

Each sample containing $50 \mu \mathrm{L}$ protein-free extract was run on a single column (ESA Meta-250, $5 \mu \mathrm{m}$ ODS, $250 \times 4.6 \mathrm{~mm}$ ID) with a 150 -minute gradient elution that ranged from $0 \%$ [Mobile Phase A consisting of $1.1 \%(\mathrm{w} / \mathrm{v})$ of 1-pentane-sulfonic acid $(\mathrm{pH}, 3.0)]$ to $20 \%$ Mobile Phase B (MPB, consisting of $0.1 \mathrm{M}$ lithium acetate in a solvent mixture of methanol, acetonitrile and isopropanol, 80/10/10 (v/v/v) with a fixed flow rate of $0.5 \mathrm{ml} / \mathrm{min}$. The temperature of both cells and column was maintained at $25^{\circ} \mathrm{C}$. Similar to the previously published method $[9,10]$, the current gradient elution also included a 2 minutes of a high-potential cell-cleaning step to restore the column to $100 \%$ MPA at the end of each run. The CMEAS is set to have increments from 0 to $960 \mathrm{mV}$ in $60 \mathrm{mV}$ steps. The properties of various antioxidant compounds or monoamine metabolites are often closely related to the same structural characteristics. The use of several coulometric sensors in series that are maintained at different potentials provides resolution and detection of co-eluting compounds with different electrochemical features.

The ESA CoulArray for Windows 32 package was used for quantitative analysis of peak identity and the peaks relative to absolute or reference standards. This software is able to automatically subtract backgrounds resulting from the gradient drift. The coulometric array is used to generate databases of all redox-active molecules with redox potentials from 0 to $960 \mathrm{mV}$. Peak identity is verified by the retention time, dominant channel, and the ratio of reactivity on the dominant channel to reactivity on the subdominant channels [9, 10]. The concentration of each peak was calculated according to the dominant channel which has $>70 \%$ ratio conformity to contribute to the quantitation [11]. In a typical assay, the standard curve for GSH and GSSG were established between 0.2 to $200 \mathrm{ng}$ that provides three orders of magnitude. Within the range of the standard curve, the coefficients of variation $(\mathrm{CV})$ for within-run assays were in the range of $0.9-6.0 \%$ and for between-run assays were in the range of 1.0-9.1\%. 


\subsubsection{Glutathione peroxidase (GPx) assay}

Approximately $200 \mathrm{mg}$ of brain tissue was homogenized in $1.5 \mathrm{ml}$ of $50 \mathrm{mM}$ Tris- $\mathrm{HCl}$ Buffer containing $1 \mathrm{mM}$ EDTA, $\mathrm{pH}$ 7.4. Following centrifugation of the homogenate for 10 minutes at $8500 \mathrm{rpm}$, aliquots of supernatant between 0.1 to $1.0 \mathrm{mg}$ proteins were used for enzyme assays.

The Cellular Glutathione Peroxidase Assay Kit (Cat. No. 354104) from Calbiochem was used to measure GPx activity indirectly. In principal, GSSG that is produced upon reduction of organic peroxide by cytoplasmic GPX is recycled to its reduced state by glutathione reductase (GR). The oxidation of NADPH to NADP ${ }^{+}$ is accompanied by a decrease in absorbance at $340 \mathrm{~nm}$, which provides a spectrophotometric means of monitoring GPx activity.

\subsubsection{Glutathione reductase (GR) assay}

The Glutathione Reductase Assay Kit from CalbIochem (Cat. No. 359962) was used to measure GR activity. Briefly, the GSSG is reduced by a multi-step reaction in which GR is initially reduced by NADPH producing a semiquinone of FAD, a sulfur radical and a thiol. The reduced GR reacts with a molecule of GSSG resulting in a disulfide interchange, which produces a

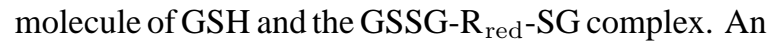
electron rearrangement in GSSG- $\mathrm{R}_{\text {red }}-\mathrm{SG}$ results in a second disulfide interchange, splitting off the second molecule of GSH and restoring the GR to the oxidized form. The GR activity was thus measured by the rate of NADPH oxidation, which is accompanied by a decrease in absorbance at $340 \mathrm{~nm}$. Since GR is present at rate-limiting concentration, the rate of decrease in the $\mathrm{A}_{340}$ is directly proportional to the GR activity in the sample.

\subsection{Statistical analyses}

Multiple regression analyses were conducted to determine whether the brain collection and storage variables (age, postmortem interval, brain weight, and storage time) were significantly associated with the biochemical measures of interest. Separate regression analyses were conducted for each of the biochemical measures. The brain collection and storage measures were entered into each of the regression analyses as predictor variables. The distributions for all variables were examined using normality plots and the KolmogorovSmimov test, which quantifies the discrepancy between data distribution and an ideal Gaussian distribution. The Dallal and Wilkinson approximation to Lilliefors' method was used to compute the $\mathrm{P}$ values. The data pass the normality test if $p>0.10$. For normally distributed data, means and variances were compared by the use of $t$-tests and $F$-tests.

One-way analyses of variance (ANOVA) were conducted to evaluate group differences for brain collection and storage parameters between schizophrenics, controls with and without other psychiatric disorders. Analyses of covariance (ANCOVA) were conducted to control statistically for the potential effects of the brain collection and storage variables on the group differences for the biochemical measures of interest. Group means for the GSH redox coupling measures were compared using ANOVAs, with pairwise comparisons (unpaired $t$-tests) following significant findings.

\section{Results}

\subsection{Brain collection and storage parameters}

One-way analyses of variance (ANOVA) were conducted to evaluate group differences for the brain collection and storage parameters (Table 1). There are no significant differences of age, postmortem interval (PMI), and storage time among three test groups with exception that brain weights was found significantly higher in schizophrenic than in control samples without psychiatric disorders. The normality plots and the Kolmogorov-Smimov test indicated that normality transformations of the data were not necessary. On the other hand, as expected, the frequency of smoking was higher in patients with either schizophrenia or bipolar and/or depression than in control subjects without psychiatric disorders. In addition, the ratio of male to female subjects was also higher in the patient samples than in control samples without psychiatric disorders.

\subsection{Evaluations of glutathione redox state}

To evaluate GSH redox state, GSH and GSSG levels were measured by HPLC coupled with a 16-channel Coulometric Electrode Array System. GSH-Px and GSSG-R activities were assayed by a spectrophotometric procedure in the caudate region of postmortem brains from schizophrenic patients and control subjects with and without other psychiatric disorders (Table 2). GSH levels were significantly $(p=0.017)$ lower in patients with schizophrenia than in control subjects without any psychiatric disorders. A similar reduction of GSH level was also found in patients with bipolar 
Table 1

Brain collection and storage parameters

\begin{tabular}{lccc} 
Parameters & Schizophrenics & \multicolumn{2}{c}{ Controls } \\
& & without PD & with other PD \\
Number of samples & 12 & 16 & 10 \\
Age, years & $60 \pm 10^{\mathrm{b}}$ & $54 \pm 17$ & $50 \pm 17$ \\
Sex (M/F) & $9 / 3$ & $10 / 6$ & $9 / 1$ \\
Smoking (yes/no) & $11 / 1$ & $10 / 6$ & $8 / 2$ \\
Postmortem interval (h) & $20 \pm 14$ & $15 \pm 7$ & $16 \pm 7$ \\
Brain weight (g) & $1404 \pm 168^{\mathrm{c}}$ & $1282 \pm 143$ & $1398 \pm 77$ \\
Storage time (months) & $17 \pm 8^{\mathrm{d}}$ & $11 \pm 8$ & $18 \pm 8$
\end{tabular}

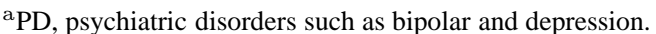

${ }^{\mathrm{b}}$ Each value represents the mean and standard deviation based on the number of examined brain samples.

${ }^{\mathrm{c}}$ ANOVA $(F=3.4482 ; d f=2.35 ; p=0.0430)$.

${ }^{\mathrm{d}}$ ANOVA $(F=2.9450 ; d f=2.45 ; p=0.0658)$.

Table 2

Evaluation of the glutathione redox system in the caudate region of postmortem brain tissue between schizophrenic and control groups

\begin{tabular}{lcccccc} 
Mercaptans & Schizophrenics & \multicolumn{2}{c}{ Controls } & \multicolumn{2}{c}{ P (two-tailed, unpaired t tests) } \\
& $(n=12)$ & without PD & with other PD & S vs C & S vs C-PD & C vs C-PD \\
& & $(n=16)$ & $(n=10)$ & & & \\
GSH $^{\mathrm{b}}$ & $107 \pm 61^{\mathrm{c}}$ & $179 \pm 89$ & $115 \pm 99$ & $\mathbf{0 . 0 1 7}$ & 0.838 & 0.110 \\
GSSG $^{\mathrm{b}}$ & $35 \pm 24$ & $33 \pm 15$ & $30 \pm 25$ & 0.870 & 0.632 & 0.670 \\
GPx & $4658 \pm 1199$ & $5825 \pm 1775$ & $6294 \pm 1210$ & $\mathbf{0 . 0 4 8}$ & $\mathbf{0 . 0 0 5}$ & 0.432 \\
GR & $685 \pm 142$ & $794 \pm 103$ & $795 \pm 218$ & $\mathbf{0 . 0 3 6}$ & 0.194 & 0.997
\end{tabular}

a Abbreviations: PD, psychiatric disorders; S, schizophrenics; C, control; C-PD, control with psychiatric disorder; GSH, glutathione; GSSG, glutathione disulfide; GPx, glutathione peroxide; GR, glutathione reductase.

${ }^{\mathrm{b}}$ Both GSH and GSSG were determined by HPLC-CMEAS procedure.

${ }^{\mathrm{c}} \mathrm{GSH}$ and GSSG were expressed as ng/mg dry wt., whereas GPx and GR were expressed as mU/mg wet wt.

and/or depression (controls with other types of psychiatric disorders) although it was not statistically significant. On the other hand, there were no significant differences of GSSG levels among the three groups of subjects. Consequently, the ratio of GSH to GSSG was significantly ( $p=0.0085$ ) lower in patients with schizophrenia than in control subjects without any psychiatric disorders (Fig. 2), but not in control subjects with bipolar and/or depression.

Both GPx and GR activities were found significantly $(p<0.05)$ lower in patients with schizophrenia than in control subjects without any psychiatric disorders (Table 2). Moreover, GPx activities in patients with schizophrenia was also significantly $(p=0.005)$ lower than that of patients with bipolar and/or depression. However, no significant differences were found in either GPx or GR between two control groups.

To further assess the GSH redox coupling reactions, levels of substrate (GSH) were correlated with the specific enzyme activities (GPx or GR). In control subjects without any psychiatric disorders, GSH levels were significantly ( $p=0.0088)$ correlated to GPx activities (Fig. 3). A similar positive correlation $(p=0.0052)$

\section{GSH:GSSG ratio}

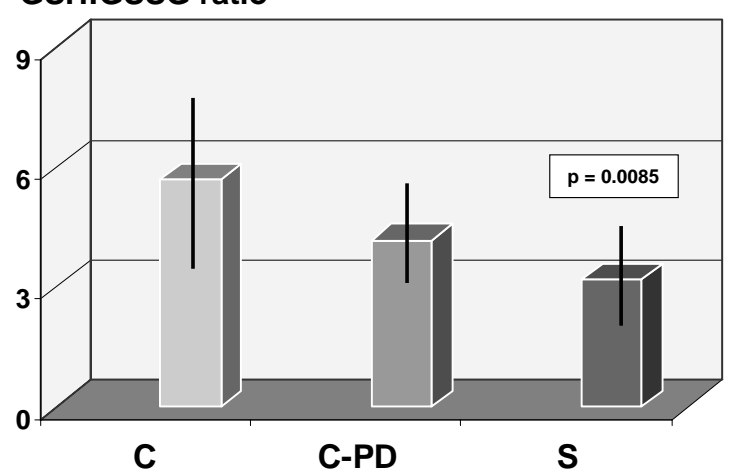

Fig. 2. Comparison of ratio of glutathione (GSH) to glutathione disulfide (GSSG) in postmortem caudates among control subjects without psychiatric disorders (C), control subjects with other psychiatric disorders (C-PD), and patients with schizophrenia (S). $p=$ 0.0085 (C vs S, two-tailed unpaired t-test).

was also demonstrated between GSH levels and GR activities (Fig. 4). Furthermore, GPx activities were significantly ( $p=0.0226)$ correlated to the GR activities in the same control group (Fig. 5). No significant correlations were found in patients with either schizophrenia 

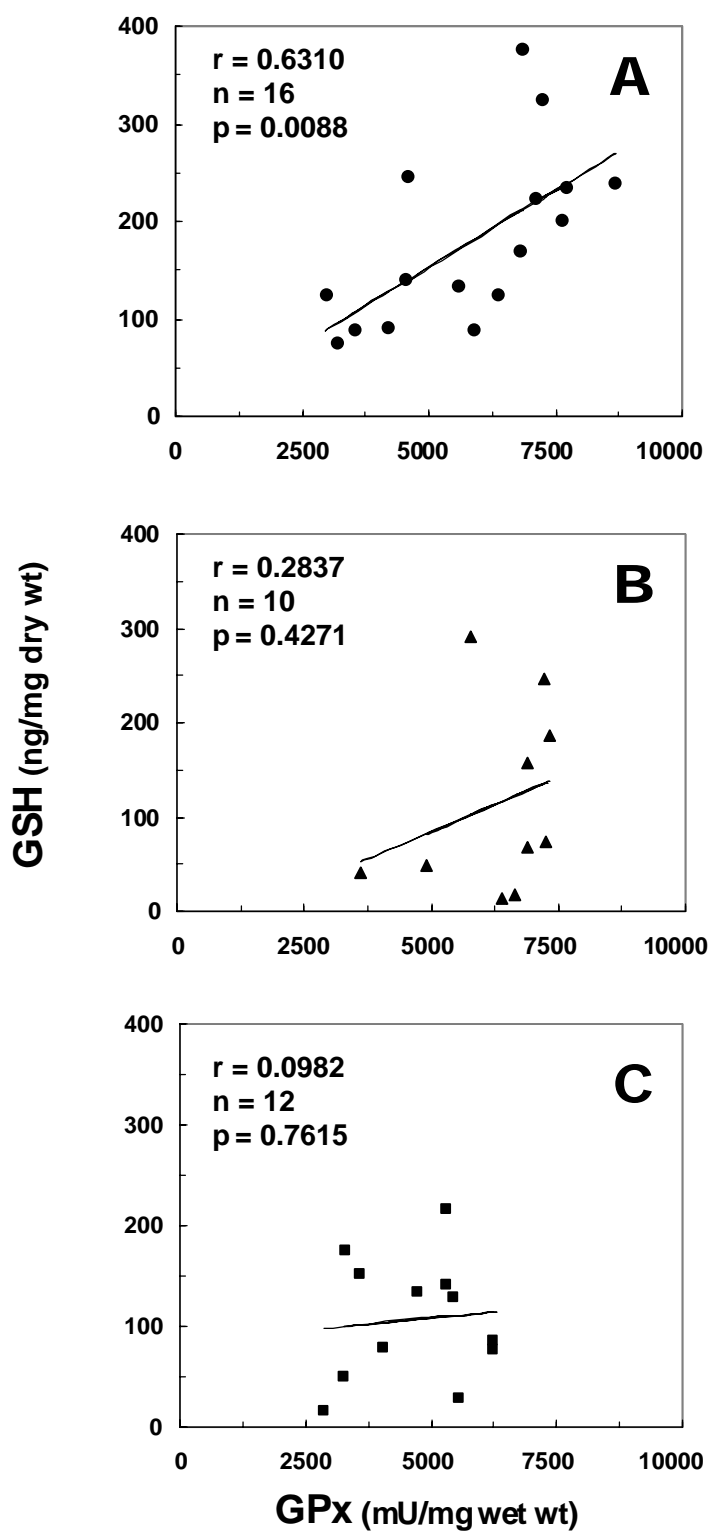

Fig. 3. Correlations of glutathione (GSH) levels to glutathione peroxidase (GPx) activities in postmortem caudate region from control subjects without psychiatric disorders (A), control subjects with bipolar and/or depression (B), and patients with schizophrenia (C).

or bipolar and/or depression (Figs 3-5).

\subsection{Effect of gender and smoking status in GSH redox state}

Although there are more female subjects in the control samples without psychiatric disorders than in patient sample groups, no significant differences of any GSH redox measures were found in control samples
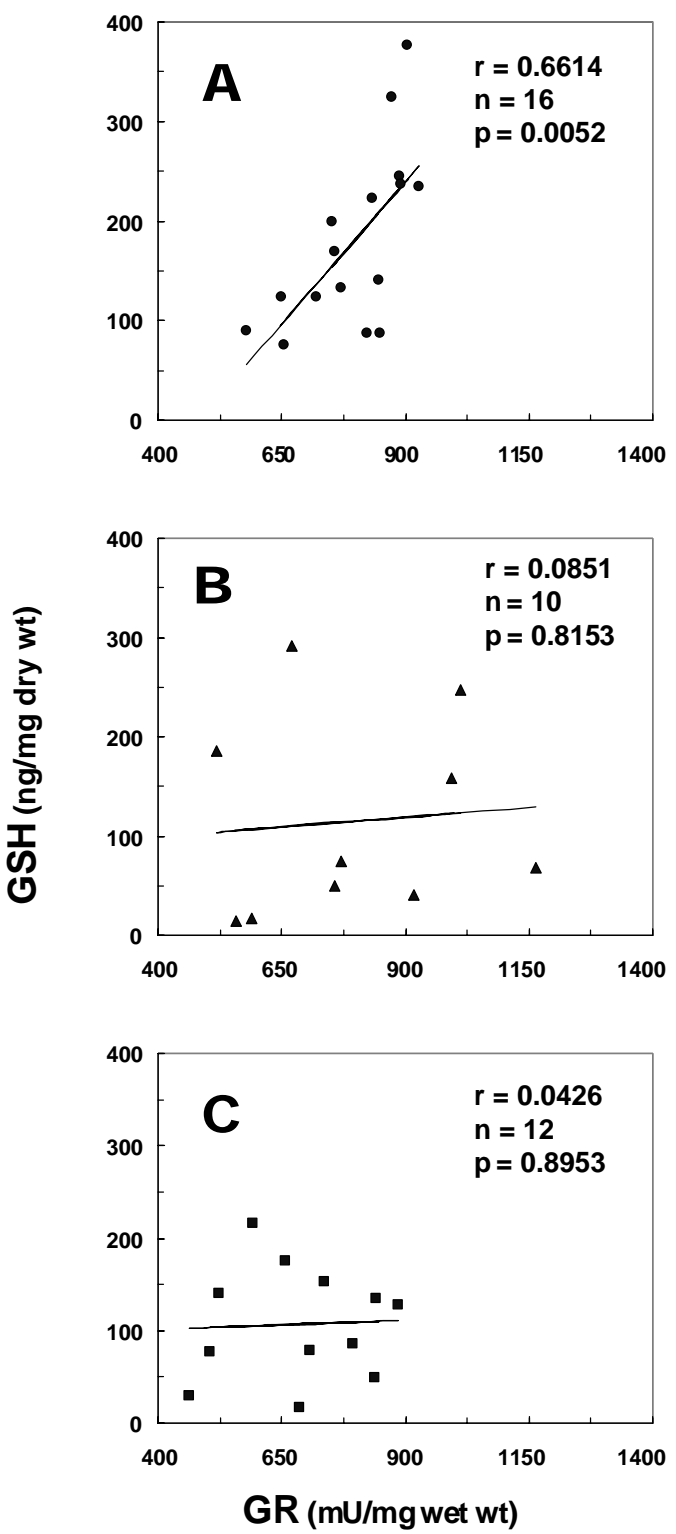

Fig. 4. Correlations of glutathione (GSH) levels to glutathione reductase (GR) activities in postmortem caudate region from control subjects without psychiatric disorders (A), control subjects with bipolar and/or depression (B), and patients with schizophrenia (C).

between female $(n=6)$ and male $(n=10)$ subjects (data not shown).

Similarly, there were no significant differences of any GSH redox measures in control samples between smokers $(n=10)$ and non-smokers $(n=10)$ in the present sample size (data not shown). When non-smokers were excluded from the three test groups, levels of GSH and GR were still found significantly lower in patients with schizophrenia than in control subjects without psy- 

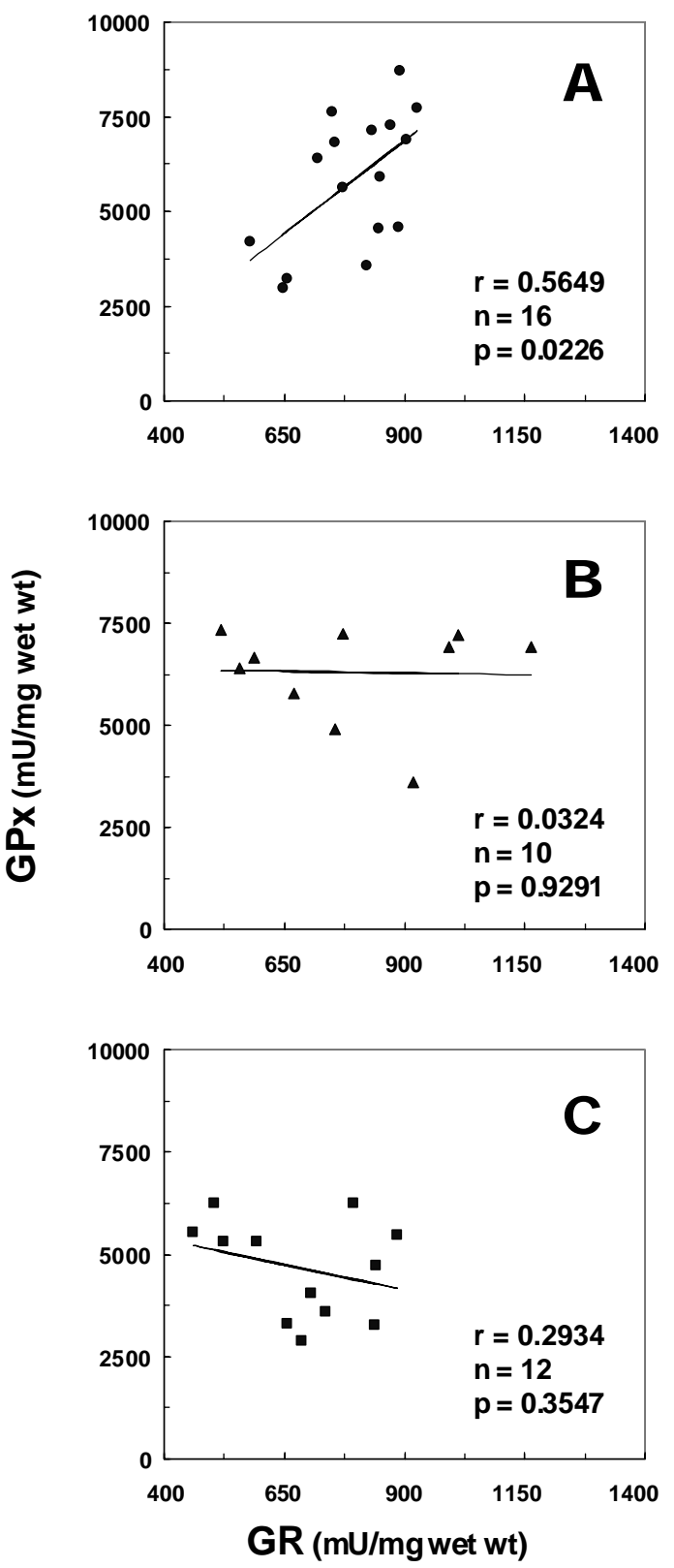

Fig. 5. Correlations of glutathione peroxidase (GPx) to glutathione reductase (GR) activities in postmortem caudate region from control subjects without psychiatric disorders (A), control subjects with bipolar and/or depression (B), and patients with schizophrenia (C).

chiatric disorders (Table 3). Likewise, GPx activities were significantly lower in patients with schizophrenia than in control subjects with bipolar and/or depression. A similar trend was also found between patients with schizophrenia and control subjects without psychiatric disorders, although the difference was not statistically significant. In addition, GSH levels were also signif- icantly lower in control subjects with bipolar and/or depression than in control subjects without psychiatric disorders (Table 3).

\subsection{Effect of age on glutathione reductase reaction}

In patients with schizophrenia, both glutathione disulfide (GSSG) and glutathione reductase (GR) were inversely correlated with age $(r=-0.6003, p=$ 0.039 ; and $r=-0.6124, p=0.0343$, respectively) in the postmortem caudate region (Figs 6 and 7). Such inverse correlations were not demonstrated in the control groups with and without other psychiatric disorders. On the contrary, GR was positively correlated with age ( $r=0.7140, p=0.0204)$ of control subjects with bipolar and/or depressive disorder (Fig. 7).

\section{Discussion}

\subsection{Quantitative determinations of GSH and GSSG}

Glutathione plays an important role in metabolism, transport, redox signaling, and cellular protection. Reduced GSH is the major non-protein thiol present in virtually all cells. Its reducing and nucleophilic properties protect cells against destructive effects of reactive oxygen species and free radicals [12]. Therefore, measurements of GSH and its metabolites provide us with useful indices in studying oxidative damage in biological samples. A recent review by Camera and Picardo [13] indicated that GSH levels reported in the literature vary more than 10 -fold mainly due to the different methodological approaches such as sample collection, processing and storage, as well as analytical instruments. To minimize GSH autooxidation and rapid enzymatic proteolysis of GSH and GSSG, it is important to store samples at $-70^{\circ} \mathrm{C}$, and process under refrigeration [13]. Although several chromatographic methods have been developed to measure GSH and GSSG, all have some disadvantages including the need to form derivatives, the inability to determine GSH and GSSG simultaneously, and a lack of detection sensitivity [14]. HPLC with electrochemical detection presents a rapid and sensitive approach to measure GSH and GSSG simultaneously $[14,15]$. However, detection of disulfides like GSSG by ECD requires application of a higher oxidation potential than GSH. In the present study, we used an HPLC-CMEAS procedure to determine levels of GSH and GSSG. The CMEAS a 16 oxidation potential range from 0 to $1000 \mathrm{mV}$. The use 
Table 3

Comparisons of glutathione redox state in the caudate region of postmortem brain tissue from smokers between schizophrenic and control groups

\begin{tabular}{lcccccc} 
Mercaptans & Schizophrenics & \multicolumn{2}{c}{ Controls } & \multicolumn{2}{c}{ P (two-tailed, unpaired t tests) } \\
& $(n=11)$ & $\begin{array}{c}\text { without PD } \\
(n=10)\end{array}$ & $\begin{array}{c}\text { with other PD } \\
(n=8)\end{array}$ & S vs C & S vs C-PD & C vs C-PD \\
GSH & $101 \pm 60^{\mathrm{b}}$ & $169 \pm 95$ & $87 \pm 84$ & $\mathbf{0 . 0 3 5}$ & 0.350 & $\mathbf{0 . 0 3 5}$ \\
GSSG & $32 \pm 23$ & $35 \pm 15$ & $31 \pm 29$ & 0.356 & 0.450 & 0.339 \\
GPx & $4782 \pm 1174$ & $5450 \pm 1612$ & $6283 \pm 1338$ & 0.149 & $\mathbf{0 . 0 1 2}$ & 0.124 \\
GR & $688 \pm 148$ & $788 \pm 104$ & $786 \pm 231$ & $\mathbf{0 . 0 4 5}$ & 0.159 & 0.490
\end{tabular}

a Abbreviations: PD, psychiatric disorders; S, schizophrenics; C, control; C-PD, control with other types of psychiatric disorder; GSH, glutathione; GSSG, glutathione disulfide; GPx, glutathione peroxide; GR, glutathione reductase.

${ }^{\mathrm{b}}$ GSH and GSSG were expressed as ng/mg dry wt., whereas GPx and GR were expressed as mU/mg wet wt.

of several coulometric sensors in series that are maintained at different potentials provides resolution and detection of co-eluting compounds such as GSH and GSSG with different electrochemical features. In addition, a filter removed macromolecules. This is considered a valid method to remove proteins because it does not require addition of acids or organic solvents that might influence sample separation, derivatization, and detection [13].

\subsection{Evaluations of GSH redox state in schizophrenia}

The present study demonstrated a significant decrease of reduced GSH in postmortem caudate from patients with schizophrenia as compared to control subjects without any psychiatric disorders (Table 2). Such a reduction was not associated with brain collection and sample storage parameters. Concomitantly, there was a significant decrease of GSH:GSSG ratio in patients with schizophrenia (Fig. 2). In cerebrospinal fluid of drug-free patients with schizophrenia, Do et al. [16] have previously demonstrated a significant decrease in the levels of GSH as compared to the controls. These same authors using a non-invasive proton magnetic resonance spectroscopy methodology also showed a similar reduction of GSH level in medial prefrontal cortex of schizophrenic patients. Our current findings are consistent with these reports and support a hypothesis of GSH deficiency in schizophrenia. The GSH deficiency induced in the animal model has been related to mitochondrial damage $[17,18]$ and increased free radical insult $[19,20]$. Moreover, several pathological conditions including Alzheimer's, Huntington's, and Parkinson's diseases have been associated with a deficiency of GSH and imbalanced ratio of GSH to GSSH [21-23].

Furthermore, the present study demonstrated positive correlations between GSH levels and GPx or GR activities in postmortem caudate from control subjects without any psychiatric disorders (Figs 3 and 4). Concomitantly, GPx was also significantly correlated with GR activities in control subjects (Fig. 5). These positive correlations suggest that a dynamic state regulates the redox coupling under normal conditions. By contrast, lack of such correlations in schizophrenia point to a disturbance of redox coupling mechanisms in the antioxidant defense system, possibly resulting from a decreased level of GSH (Table 2) as well as age-related decreases of GSSG and GR activities (Figs 6 and 7).

\subsection{Antipsychotics and the GSH redox state}

It is possible that alterations in the GSH redox state is attributable to the long-term treatment with antipsychotic treatment that these patients likely received. In the present study, all subjects except control subjects without any psychiatric disorders were on antipsychotic medications at the time of death. No significant differences, however, were demonstrated in the caudate mercaptans between control subjects with or without non-schizophrenic psychiatric disorders.

On the other hand, GSH plays a pivotal role in protecting cells from oxidative damage such as induced by dopamine (DA) oxidation. Recently, Grima et al. [24] have proposed that GSH deficit in schizophrenia may be associated with DA-induced oxidative stress. Antipsychotic drugs are known to block DA receptors and consequently, may result in increased levels of GSH. Therefore, the decreased GSH levels observed in this study are unlikely due to antipsychotic effects, because GSH levels may be evenly lower in patients with schizophrenia or other psychoses in the absence of antipsychotic treatment.

\subsection{Effect of cigarette smoking on GSH redox state}

The prevalence of cigarette smoking in schizophrenic patients is between $70 \%-90 \%$, compared to that of 35- 

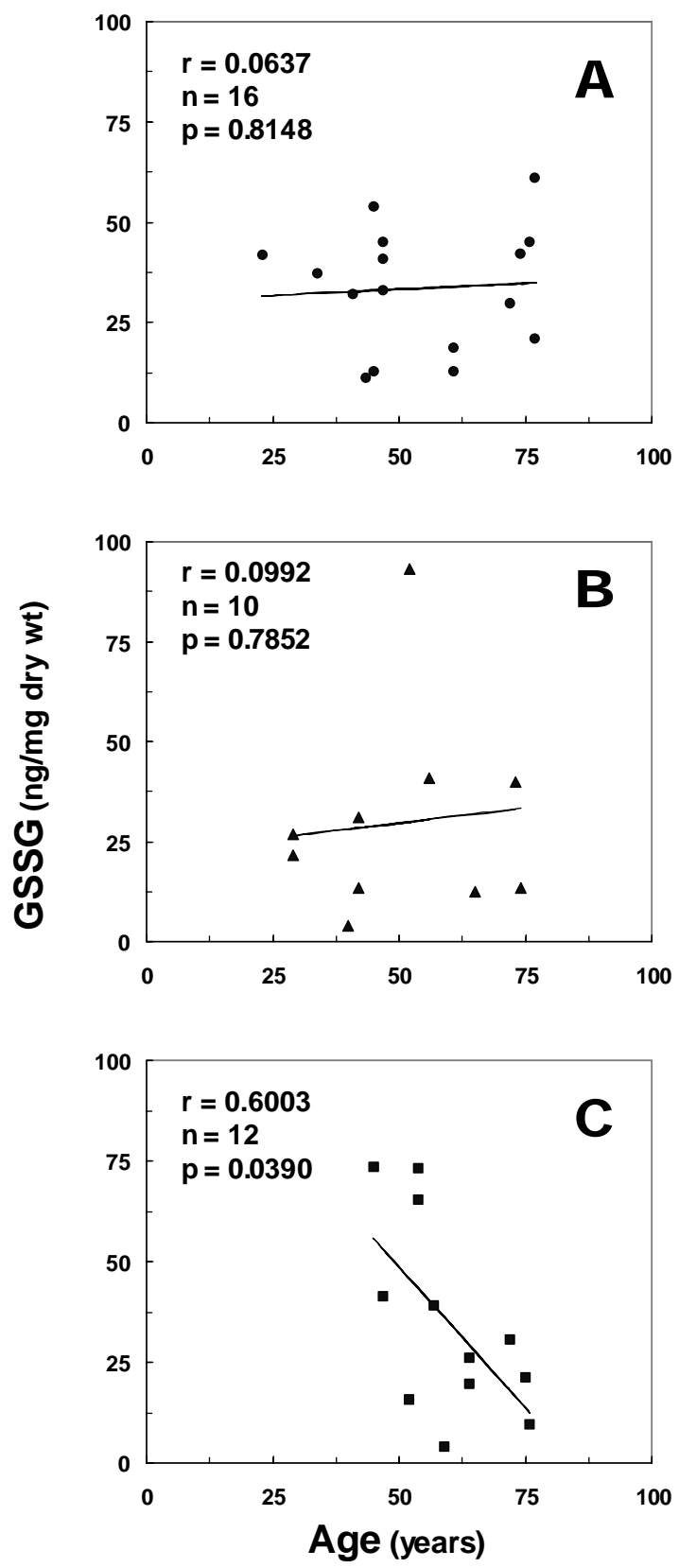

Fig. 6. Correlations of glutathione disulfide (GSSG) levels to age in postmortem caudate region from control subjects without psychiatric disorders (A), control subjects with bipolar and/or depression (B), and patients with schizophrenia (C).

$54 \%$ for all psychiatric patients and $30-35 \%$ for general population [25-27]. Cigarette smoke contains, in addition to nicotine, thousands of substances in the gas and tar phases, many of which are free radicals such as peroxyl radicals, oxides of nitrogen, hydroquinones and other radical species [28-31]. Several studies have
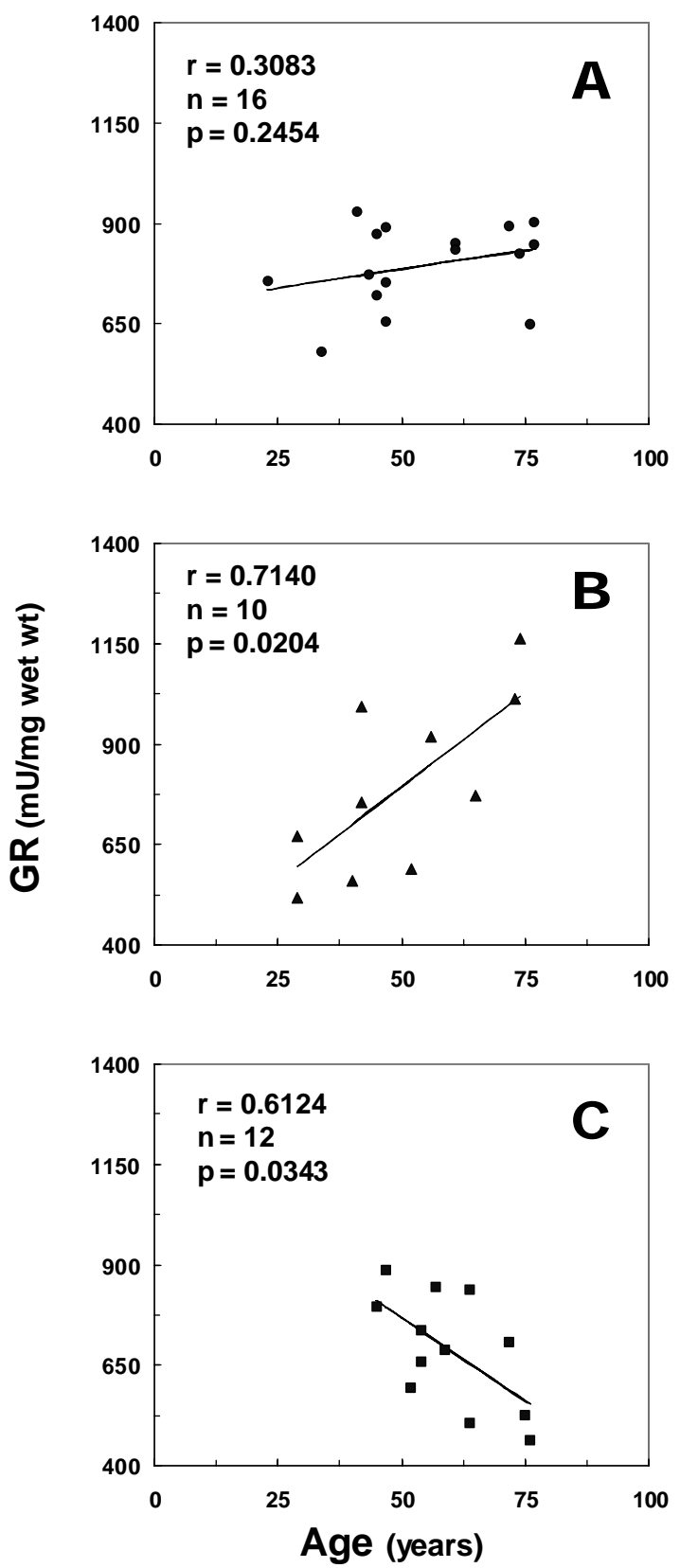

Fig. 7. Correlations of glutathione reductase (GR) activities to age in postmortem caudate region from control subjects without psychiatric disorders (A), control subjects with bipolar and/or depression (B), and patients with schizophrenia (C).

shown that exposure of isolated cells such as epithelial cells and neutrophils or cultured cells to cigarette smoke can lead to rapid depletion of intracellular GSH levels [32-34]. Cigarette smoke induces cell permeability $[35,36]$ and oxidation of lipids and proteins [37, 38] can be prevented by a low-molecular weight an- 
tioxidant such as GSH.

As expected, the majority of samples from schizophrenic or bipolar and/or depression groups were obtained from smokers (Table 1). However, the reduced GSH levels found in both patient groups do not appear to be related to cigarette smoking (Table 3 ).

\subsection{GSH redox coupling and reactive nitrogen species}

Nitric oxide (NO) is known to have an inhibitory effect on mitochondrial respiration. Following exposure to nitric oxide, GSH will react with $\mathrm{NO}^{-}$to form hydroxylamine and oxidized GSH (GSSG), and with NO+ to produce S-nitrosoglutathione [39]. Moreover, peroxynitrite will also react with GSH via either a one or two electron oxidation process, depending on the physiological $\mathrm{pH}$ and GSH concentrations [40]. Thus, the intracellular GSH concentration appears to be a critical factor in dictating susceptibility to NO and its derivatives [41]. Under physiologic conditions, NO and its metabolites react with a variety of thiol compounds to form dissociable complexes [42], thereby regulating its inhibitory function [43]. We have recently demonstrated a significantly increased level of NO in the caudate region of postmortem brain from these same individuals with schizophrenia [44], supporting the notion of free radical-mediated neurotoxicity in schizophrenia. Because the reaction of NO with free thiols competes with the same substrate such as glutathione (GSH) for decomposition of hydrogen peroxide by GSH peroxidase, the excessive NO formation may further lead to significant depletion of GSH in schizophrenia.

\section{Acknowledgements}

This work was supported in part by the grants from Office of Research and Development, Department of Veterans Affairs [Merit Reviews (JKY, SL) and Research Career Scientist Award (JKY, SL)], National Institute of mental Health [MH58141 (JKY), MH64118 (RDR)], DA09457 (SL), NARSAD Young Investigator Award (RDR), NARSAD Independent Investigator Award (SL), and the Highland Drive VA Pittsburgh Healthcare System. The authors are grateful to B. Bland, P. Cheng and B. Sullivan for their technical assistance.

\section{References}

[1] H.J. Forman, J.M. Fukuto and M. Torres, Redox signaling: thiol chemistry defines which reactive oxygen and nitrogen species can act as second messengers, Am J Physiol Cell Physiol 287 (2004), 246-256.

[2] J.L. Cadet and J.B. Lohr, Free radicals and the developmental pathology of schizophrenic burnout, Integrative Psychiatry 5 (1987), 40-48.

[3] S. Mukherjee, S.P. Mahadik, R. Scheffer, E.E. Correnti and H. Kelkar, Impaired antioxidant defense at the onset of psychosis, Schizophr Res 19 (1996), 19-26.

[4] J.K. Yao, R.D. Reddy and D.P. van Kammen, Oxidative Damage and Schizophrenia: An Overview of the Evidence and Its Therapeutic Implications, CNS Drugs 15 (2001), 287-310.

[5] J.K. Yao and P. Cheng, Determination of multiple redoxactive compounds by high-performance liquid chromatography with coulometric multi-electrode array system, J. Chromatogr B810 (2004), 93-100.

[6] American Psychiatric Association, Diagnostic and Statistical Manual of Mental Disorders, (Fourth ed.), Washington, DC: American Psychiatric Association, 1994.

[7] J.A. Hardy, P. Wester, B. Winblad, C. Gezelius, G. Bring and A. Eriksson, The patients dying after long terminal phase have acidotic brains; implications for biochemical measurements on autopsy tissue, J Neural Transm 61 (1985), 253-264.

[8] P.J. Harrison, P.R. Heath, S.L. Eastwood, P.W.J. Burnet, B. McDonald and R.C.A. Pearson, The relative importance of premortem acidosis and postmortem interval for human brain gene expression studies: Selective mRNA vulnerability and comparison with their encoded proteins, Neuroscience Letters 200 (1995), 151-154.

[9] P.E. Milbury, CEAS Generation of large multi-parameter metabolic databases for determining categorical process involvement of biological molecules, in: Progress in HPLCHPCE, (Vol. 6), I.N. Acworth et al., eds, VSP International Science Publication, Utrecht, Holland, 1997, pp. 127-144.

[10] B.S. Kristal, K. Vigneau-Callahan and W.R. Matson, Simultaneous analysis of multiple redox-active metabolites from biological matrices, in: Methods in Molecular Biology, (Vol. 186), Oxidative Stress Biomarkers and Antioxidant Protocols, D. Armstrong, ed., Humana Press Inc., Totowa, NJ, 2002, pp. 185-194.

[11] H. Shi, K.E. Vigneau-Callahan, W.R. Matson and B.S. Kristal, Attention to relative response across sequential electrodes improves quantitation of coulometric array, Anal Biochem $\mathbf{3 0 2}$ (2002), 239-245.

[12] A. Meister, Selective modification of glutathione metabolism, Science 220 (1983), 472-477.

[13] E. Camera and M. Picardo, Analytical methods to investigate glutathione and related compounds in biological and pathological processes, J Chromatography B781 (2002), 181-206.

[14] J. Lakritz, C.G. Plopper and A.R. Buckpitt, Validated highperformance liquid chromatography-electrochemical method for determination of glutathione and glutathione disulfide in small tissue samples, Anal Biochem 247 (1997), 63-68.

[15] N.C. Smith, M. Dunnett and P.C. Mills, Simultaneous quantitation of oxidized and reduced glutathione in equine biological fluids by reverse-phase high-performance liquid chromatography using electrochemical detection, J Chromatogr B Biomed Appl 673 (1995), 35-41.

[16] K.Q. Do, A.H. Trabesinger, M. Kirsten-Kruger, C.J. Lauer, U. Dydak, D. Hell, F. Holsboer, P. Boesiger and M. Cuenod, Schizophrenia: glutathione deficit in cerebrospinal fluid and 
prefrontal cortex in vivo, Eur J Neurosci 12 (2000), 37213728.

[17] A. Meister, Mitochondrial changes associated with glutathione deficiency, Biochim Biophys Acta 1271 (1995), 35-42.

[18] T.A. Seaton, P. Jenner and C.D. Marsden, Mitochondrial respiratory enzyme function and superoxide dismutase activity following brain glutathione depletion in the rat, Biochem Pharmacol 52 (1996), 1657-1663.

[19] M.E. Anderson, Glutathione: an overview of biosynthesis and modulation, Chem Biol Interact 111-112 (1998), 1-14.

[20] J. Navarro, E. Obrador, J.A. Pellicer, M. Aseni, J. Vina and J.M. Estrela, Blood glutathione as an index of radiationinduced oxidative stress in mice and humans, Free Radic Biol Med 22 (1997), 1203-1209.

[21] N. Jha, O. Jurma, G. Lalli, Y. Liu, E.H. Pettus, J.T. Greenamyre, R.-M. Liu, H.J. Forman and J.K. Andersen, Glutathione Depletion in PC12 Results in Selective Inhibition of Mitochondrial Complex I Activity. IMPLICATIONS FOR PARKINSON'S DISEASE, J Biol Chem 275 (2000), 2609626101.

[22] J.D. Adams Jr, L.K. Klaidman, M.L. Chang and J. Yang, Brain oxidative stress - analytical chemistry and thermodynamics of glutathione and NADPH, Curr Top Med Chem 1 (2001), 473-482.

[23] J. Keelan, N.J. Allen, D. Antcliffe, S. Pal and M.R. Duchen, Quantitative imaging of glutathione in hippocampal neurons and glia in culture using monochlorobimane, $J$ Neurosci Res 66 (2001), 873-884.

[24] G. Grima, B. Benz, V. Parpura, M. Cuenod and K.Q. Do, Dopamine-induced oxidative stress in neurons with glutathione deficit: implication for schizophrenia, Schizophr Res 62 (2003), 213-224.

[25] J.R. Hughes, D.K. Hatsukami, J.E. Mitchell and L.A Dahlgren, Prevalence of smoking among psychiatric outpatients, Am J Psychiatry 143 (1986), 993-997.

[26] J.B. Lohr and K. Flynn, Smoking and schizophrenia, Schizophr Res 8 (1992), 93-102.

[27] A. Olincy, D.A. Young and R. Freedman, Increased levels of the nicotine metabolite cotinine in schizophrenic smokers compared to other smokers, Biol Psychiatry 42 (1997), 1-5.

[28] D.F. Church and W.A. Pryor, Free radical chemistry of cigarette smoke and its toxicological implications, Environ Health Perpect 64 (1985), 111-126.

[29] K.C. Chow, Vitamin E and cigarette smoking-induced oxidative damage, in: Vitamin E in Health and Disease, L. Packer and J. Fuchs, eds, Marcel Decker, New York, 1992, pp. 683697.

[30] W.A. Pryor and K. Stone, Oxidants in cigarette smoke. Radicals, hydrogen peroxide, peroxynitrate, and peroxynitrite, Ann N Y Acad Sci 686 (1993), 12-27.

[31] B. Stegmayr, I. Johansson, F. Huhtasaari, U. Moser and K. Asplund, Use of smokeless tobacco and cigarettes - Effects on plasma levels of antioxidant vitamins, Int J Vitm Nutr Res 63 (1993), 195-200.

[32] H. Nguyen, E. Finkelstein, A. Reznick, C. Cross and A. van der Vliet, Cigarette smoke impairs neutrophil respiratory burst activation by aldehyde-induced thiol modifications, Toxicology 160 (2001), 207-217.

[33] I. Rahman and W. MacNee, Lung glutathione and oxidative stress: implications in cigarette smoke-induced airway disease, Am J Physiol Lung Cell Mol Physiol 277 (1999), 10671088.

[34] C. Piperi, A.E. Pouli, N.A. Katerelos, D.G. Hatzinikolaou, A. Stavridou and M.C. Psallidopoulos, Study of the mechanisms of cigarette smoke gas phase cytotoxicity, Anticancer Res $\mathbf{2 3}$ (2003), 2185-2190.

[35] J. Nagy, E.G. Demaster, I. Wittmann, P. Shultz and L. Raij, Induction of endothelial cell injury by cigarette smoke, Endothelium 5 (1997), 251-263.

[36] C. Rusznak, R.J. Sapsford, J.L. Devalia, R.J. John, E.L. Hewitt, A.G. Lamont, A.J. Wood, S.S. Shah, R.J. Davies and S. Lozewicz, Cigarette Smoke Potentiates House Dust Mite Allergen-Induced Increase in the Permeability of Human Bronchial Epithelial Cells In Vitro, Am J Respir Cell Mol Biol 20 (1999), 1238-1250.

[37] C.E. Cross, C.A. O’Neill, A.Z. Reznick, M.L. Hu, L. Marcocci, L. Packer and B. Frei, Cigarette smoke oxidation of human plasma constituents, Ann NY Acad Sci 686 (1993), $72-89$.

[38] J.P. Eiserich, A. van der Vliet, G.J. Handelman, B. Halliwell and C.E. Cross, Dietary antioxidants and cigarette smokeinduced biomolecular damage: a complex interaction, $A m J$ Clinical Nutrition 62 (1995), 1490-1500.

[39] M.N. Hughes, Relationships between nitric oxide, nitroxyl ion, nitrosonium cation and peroxynitrite, Biochim Biophys Acta 1411 (1999), 263-272.

[40] C. Quijano, B. Alvarez, R.M. Gatti, O. Augusto and R. Radi, Pathways of peroxynitrite oxidation of thiol groups, Biochem J 322 (1997), 167-173.

[41] J.P. Bolanos, S.J. Heales, S. Peuchen, J.E. Barker, J.M. Land and J.B. Clark, Nitric oxide-mediated mitochondrial damage: a potential neuroprotective role for glutathione, Free Radic Biol Med 21 (1996), 995-1001.

[42] V.G. Kharitonov, A.R. Sundquist and V.S. Sharma, Kinetics of nitrosation of thiols by nitric oxide in the presence of oxygen, J Biol Chem 270 (1995), 28158-28164.

[43] S.J. Heales and J.P. Bolanos, Impairment of brain mitochondrial function by reactive nitrogen species: the role of glutathione in dictating susceptibility, Neurochem Int 40 (2002), $469-474$.

[44] J.K. Yao, S. Leonard and R.D. Reddy, Increased nitric oxide radicals in postmortem brain from patients with schizophrenia, Schizophr Bull 30 (2004), 923-934. 


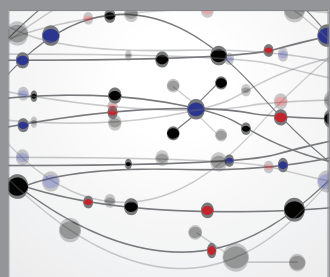

The Scientific World Journal
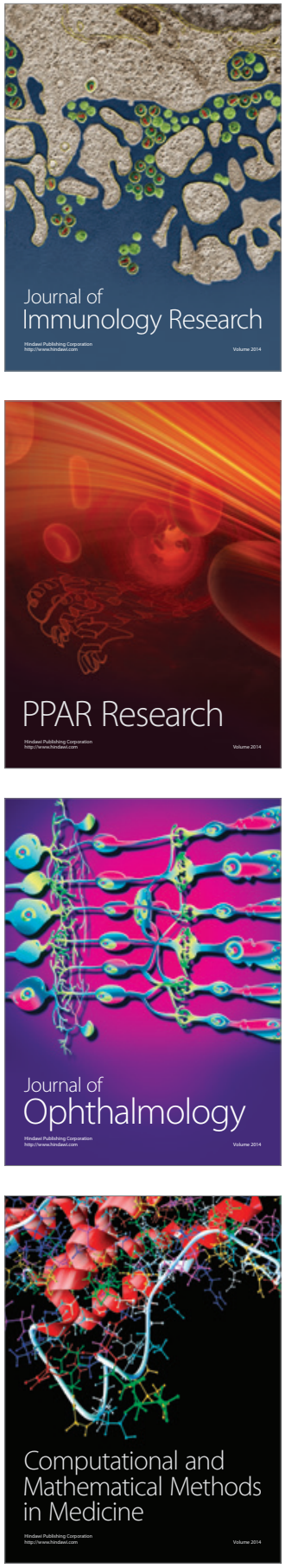

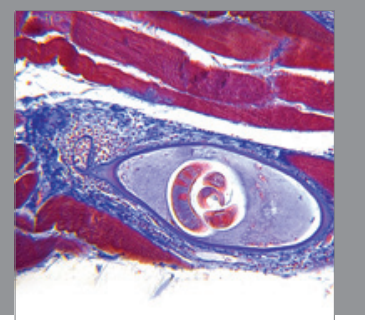

Gastroenterology

Research and Practice
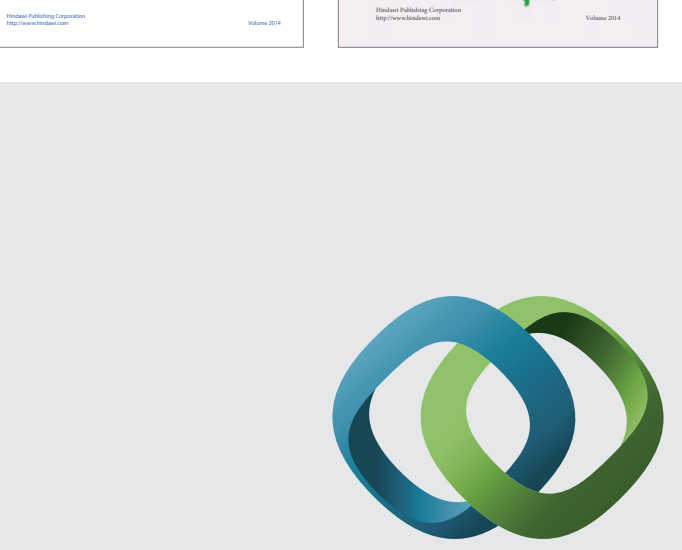

\section{Hindawi}

Submit your manuscripts at

http://www.hindawi.com
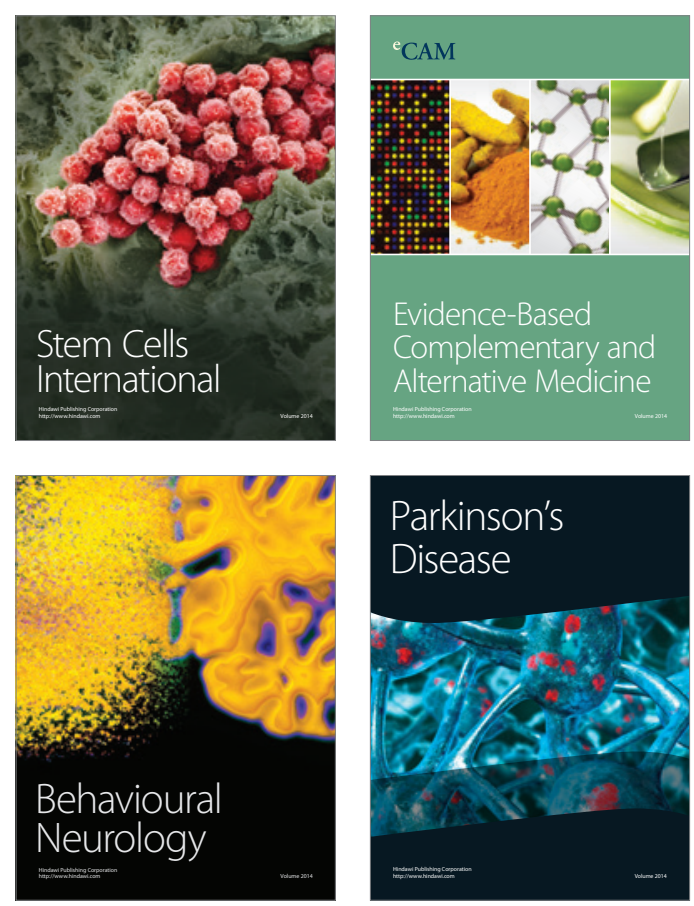

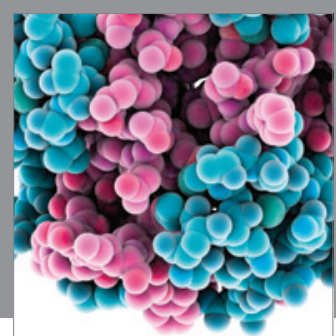

Journal of
Diabetes Research

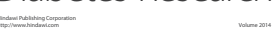

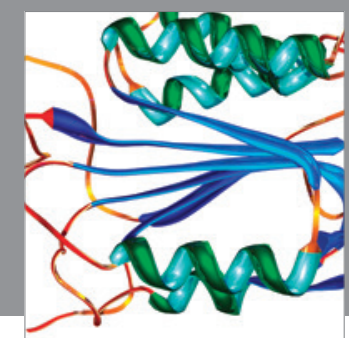

Disease Markers
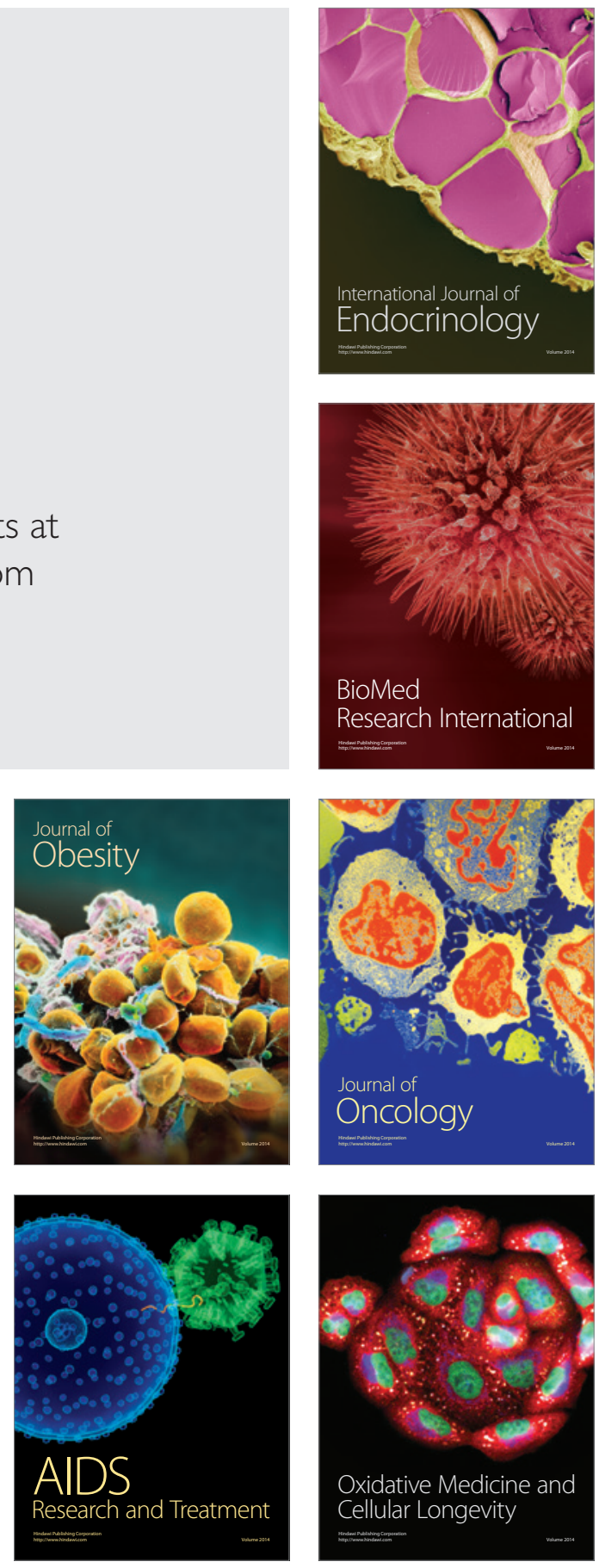